\section{Ball Lightning}

This communication records the observation of ball lightning in unusual circumstances. I was seated near the front of the passenger cabin of an all-metal airliner (Eastern Airlines Flight EA 539) on a late night flight from New York to Washington. The aircraft encountered an electrical storm during which it was enveloped in a sudden bright and loud electrical discharge $(0005 \mathrm{~h}$ EST, March 19, 1963). Some seconds after this a glowing sphere a little more than $20 \mathrm{~cm}$ in diameter emerged from the pilot's cabin and passed down the aisle of the aircraft approximately $50 \mathrm{~cm}$ from me, maintaining the same height and course for the whole distance over which it could be observed.

The observation was remarkable for the following reasons. (i) The appearance of the phenomenon in an almost totally screened environment; (ii) the relative velocity of the ball to that of the containing aircraft was $1.5 \pm 0.5 \mathrm{~m} \mathrm{~s}^{-1}$, typical of most ground observations; (iii) the object seemed perfectly symmetrical in all three dimensions and had no polar or torroidal structure; (iv) it was slightly limb darkened having an almost solid appearance and indicating that it was optically thick; (v) the object did not seem to radiate heat; (vi) the optical output could be assessed as approximately 5 to $10 \mathrm{~W}$ and its colour was blue-white; (vii) the diameter was $22 \pm 2$ $\mathrm{cm}$, assessed by eye relative to the surroundings; (viii) the height above the floor was approximately $75 \mathrm{~cm}$; (ix) the course was straight down the whole central aisle of the aircraft; ( $\mathrm{x}$ ) the object seemed to be in perfect equilibrium; (xi) the symmetry of the object was such that it was not possible to assess whether or not it was spinning.

It is not easy to reconcile the symmetry of the ball and the lack of radiant heat with many of the theories that have been proposed for ball lightning1-5, though these aspects of the observation are consistent with many other cases reported by Singer ${ }^{6}$.

\section{R. C. JENNISON}

Electronics Laboratories,

University of Kent,

Canterbury, Kent.

Received August 25, 1969.

${ }^{3}$ Bruce, C. E. R., Nature, 202, 996 (1964).

2 Kapitz, P. L., Dokl., USSR (1955).

${ }^{3}$ Lindberg, L., Witalis, E., and Jacobson, C. T., Nature, 185, 452 (1960).

- Watson, W. K. R., Nature, 185, 449 (1960).

${ }^{5}$ Wooding, E. R., Nature, 199, 272 (1963).

- Singer, S., Nature, 198, 745 (1963).

\section{Detonative Phase Transformations and Earthquakes}

IN detonation, the energy liberated supports a shock front the impact of which on as yet unreacted material induces further energy release progressively. Elementary hydrodynamic theory suggests that it should be possible for a wide variety of physico-chemical reactions to be propagated in this way, at their characteristic detonation rates. But in practice detonation has been observed only for comparatively very few systems. The main reason for the (fortunate) rarity of detonations in physico-chemical reactions is that unless its liberation is very rapid, most of the energy of reaction can be dissipated sideways, instead of contributing to the hydrodynamic propagation of the shock front, the impact of which has in turn to trigger transformation in unreacted material ahead of it. Normally as a result of lateral energy losses, only very intense reactions can establish a detonation front with sufficient stability to be identifiable as such in the laboratory, or on the ordinary technological scale.
But when the dimensions of the reaction system become greatly enlarged, for example on the geological scale, energy dissipation for even weak detonations becomes relatively much less important ${ }^{1}$. Weak detonations are thus a definite possibility for really large masses of reacting material. Tentative dimensional conditions for stability of detonation can be written down from general similitude considerations ${ }^{1}$, though direct proof that certain earthquakes originate from solid-solid phase transformations or other reactions in the condensed state proceeding at rates appropriate to weak detonation is difficult to establish in normal conditions. Velocities of propagation of weak detonations must in any case lie close to velocities of propagation of elastic displacements; only integral evaluation of the total energy liberated in any normal earthquake might disprove or permit its definite attribution to phase transformations on a geological scale.

With the advent of seismic studies on underground nuclear explosions ${ }^{2}$, new possibilities of testing such attribution become feasible. "Secondary" earthquakes resulting from nuclear explosions can be envisaged (i) through the triggering of transformations at detonative rates in thermodynamically unstable phases already present in large masses initially; (ii) less probably, by the action of the primary shock causing transformation of fairly large masses into an unstable solid state, which relapses spontaneously as a secondary earthquake only after some lapse of time.

Repeated underground nuclear explosions in the same area would eventually exhaust (i), whereas (ii) could permit indefinite recurrence of secondary localized earthquakes wherever the geochemistry favoured the necessary underlying physico-chemical changes due to the primary shock wave. Evidence about secondary earthquakes from nuclear explosions ${ }^{2}$ does not yet extend to a sufficient diversity of geochemistries to permit definite conclusions about the eventual exhaustion of seismic sensitivity, and thus to support (i). Nevertheless, the possibilities of weak detonations on a geological scale may now be closer to practical realization and should be kept in mind.

Department of Chemical Engineering

A. R. UBBELOHDE

and Chemical Technology;

Imperial College,

London SW7.

Received August 19, 1969.

1 Ubbelohde, A. R., Proc. Roy, Soc, 204, A, 25 (1950); Fourth Intern. Symp.

on Combustion, 464 (Williams and Wilkins, Baltimore, 1963)

2 Boucher, G., Ryall, A., and Jones, A. E., J. Geophys. Res., 74, 3808 (1969).

\section{Polarization Characteristics of Individual Elements in a Pc I Micropulsation Event}

Polarization characteristics of Pc 1 micropulsations of the Earth's magnetic field were examined in narrow frequency bands. Wide-band observations were reported earlier ${ }^{1}$. The induction magnetometer data were recorded at College, Alaska, in FM mode on magnetic tape. The data were played back through a bank of active band-pass filters into a dual-beam oscilloscope. Each filter had attenuation slope $24 \mathrm{~dB} /$ octave nominal value and approximately $12 \mathrm{~dB} /$ octave at a cut-off. Two filters were used in series for each magnetic field component. The high-frequency and low-frequency cut-offs were set equal to the desired mid-band frequency $f_{m}$, then the filters were tuned to preserve the shape and sense of rotation for test patterns at $f_{m}$. There was considerable phase shift through the filters, but this procedure preserved the shape 